\title{
Coupled-wave theory of multiple-stripe semiconductor injection lasers
}

\author{
David Mehuys and Amnon Yariv \\ Department of Applied Physics, 128-95, California Institute of Technology, Pasadena, California 91125
}

Received February 3, 1988; accepted April 24, 1988

\begin{abstract}
We apply coupled-wave theory to describe the lateral modes of semiconductor lasers with a periodic gain and refractive-index variation across their widths. The model is relevant to devices whose complex index of refraction is determined by current injection from closely spaced parallel electrodes. Good agreement is observed between the analytical modes and those computed numerically for comparison.
\end{abstract}

By design, semiconductor laser arrays consist of a number of single-mode waveguides placed in close proximity, so that distributed coupling occurs through modal overlap. The lateral modes of the array are ideally described by superposition of the modes of the uncoupled waveguides, or supermodes. ${ }^{1,2}$ Indeed, arrays formed from index-guided elements are accurately modeled by using supermode (i.e., coupled-mode) theory. However, arrays formed from gain-guided elements, such as oxide-isolated or proton-bombarded stripes, are not. Since the interelement coupling is inherently stronger than coupling in the index-guided case, ${ }^{3,4}$ the modes of individual gain-guided waveguides do not constitute a good basis from which to construct the lateral modes of the composite waveguide. Numerical modeling predicts mode numbers greater than the number of array elements. ${ }^{5,6}$ Furthermore, with the advent of injection-seeding techniques ${ }^{5}$ and external grating-tuned cavities, ${ }^{7}$ the existence of these higher-order modes has been verified experimentally.

In this Letter, we describe an analytical model of multiple-stripe lasers based on laterally counterpropagating plane waves that are coupled by the periodic gain and index variation across the device width. The coupling is similar to that within distributed-feedback lasers, ${ }^{8}$ except that here the feedback takes place in the lateral direction rather than along the laser axis. In addition to describing the low-order modes $(\nu \leq N)$, our model correctly predicts the existence of lateral modes of orders higher $(\nu>N)$ than the number of injection electrodes $N$. Furthermore, these higherorder modes agree well with those found experimentally by injection seeding. ${ }^{5}$

The waveguide prototype for this research is shown in the inset of Fig. 1; it is a 10-stripe laser with $4.5-\mu \mathrm{m}$ wide stripes situated on $9-\mu \mathrm{m}$ centers. An antiguiding factor of 1.5 and an outer loss of $85 \mathrm{~cm}^{-1}$ are assumed; we estimate these to be appropriate for the lowthreshold single-quantum-well wafers grown in our laboratory by molecular-beam epitaxy. The interchannel gain of $0 \mathrm{~cm}^{-1}$ is intended to model devices with shallow (if any) proton implantation-this model can be inferred from spontaneous emission profiles ${ }^{7}$ or from diffraction effects in diode-array traveling-wave amplifiers. ${ }^{9}$

Figure 1 plots the eigenvalues (mode index and modal gain) for the first 14 modes of this waveguide, as obtained by direct numerical integration of the wave equation. While the $\nu=10$ mode has the highest modal gain, the next-highest modal gains belong to the $\nu=11$ and $\nu=12$ modes. This shows the inadequacy of supermode theory as applied to gain-guided devices, since modes with $\nu>10$ cannot be accounted for. Also note that the gains of the first 14 modes exceed the interchannel gain. Hence, even if the gain outside the laser matched the interchannel gain (as in a deeply proton-implanted laser), the cutoff would be greater than $\nu=14$ (this has been verified numerically). The near-field and far-field intensities of selected modes are shown as solid lines in Figs. 2 and 3.

We begin the theoretical analysis by assuming traveling waves in the $+z$ direction of the form $E(x) \exp \left(i k_{0} \eta z\right)$, where $x$ is the lateral dimension, $k_{0}=$ $2 \pi / \lambda_{0}$ is the free-space propagation constant, and $k_{0} \eta$

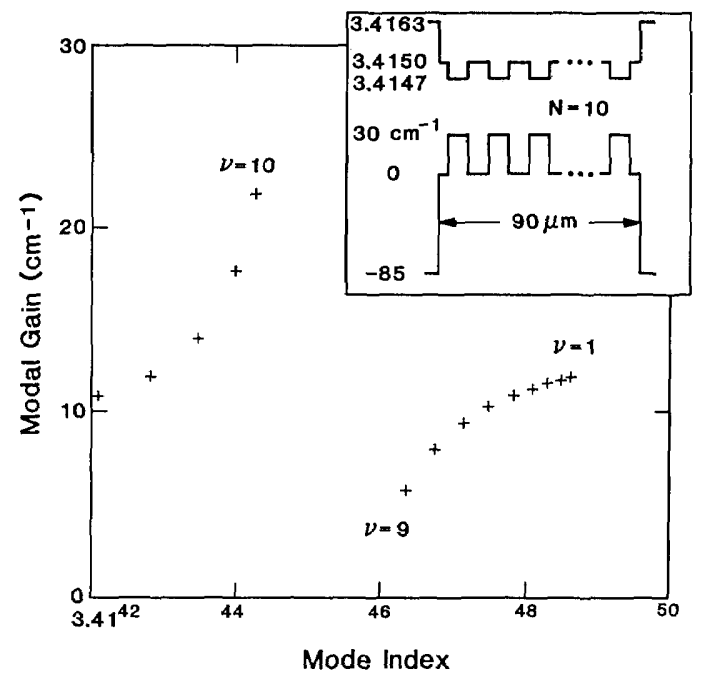

Fig. 1. Plot of the lowest 14 eigenvalues (mode index and modal gain) found by numerical integration for the waveguide shown in the inset. 

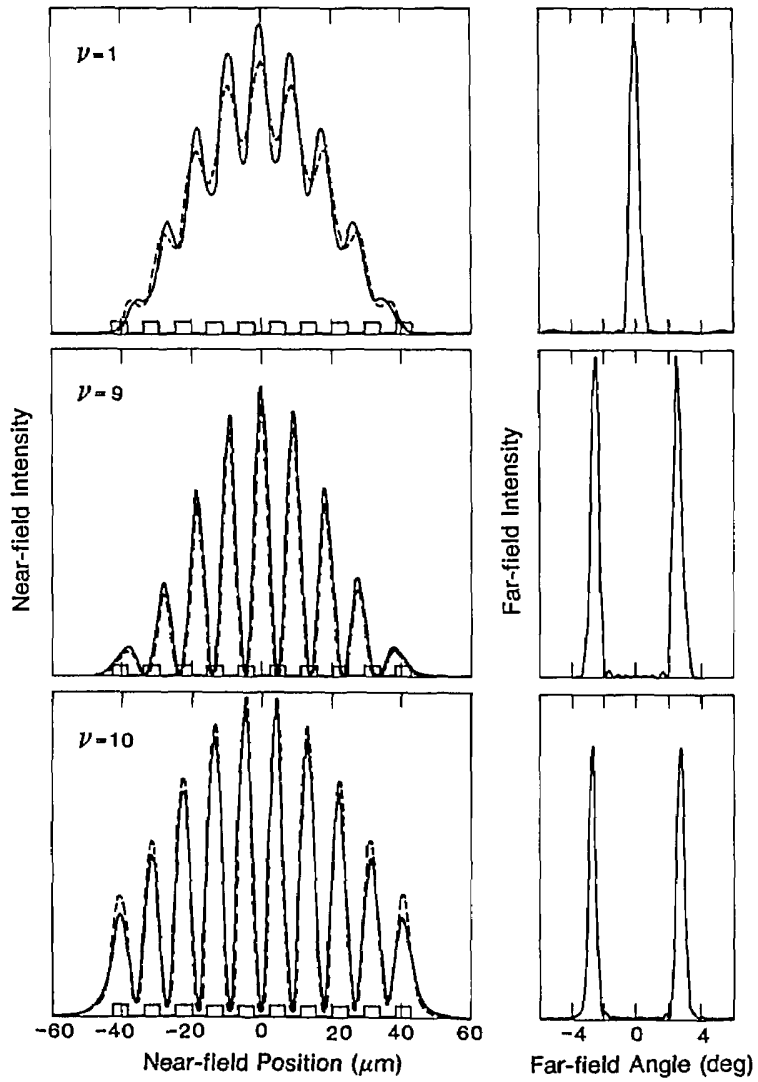

Fig. 2. Near-field and far-field intensities for the $\nu=1,9$, and 10 lateral modes found by numerical solution (solid lines) compared with the analytical solution (dashed lines).

is the (unknown) propagation constant of the lateral mode $E(x)$. To model the multiple-stripe laser, we take an effective index distribution

$$
n^{2}(x)=\left\{\begin{array}{ll}
n_{0}{ }^{2}+2 n_{1}{ }^{2} \cos (4 \pi x / \Lambda) & |x|<x_{0} \\
\bar{n}_{0}{ }^{2} & |x| \geq x_{0}
\end{array},\right.
$$

so that $\Lambda / 2$ is the period of the perturbation, $2 n_{1}^{2}$ is its amplitude, and $\bar{n}_{0}$ is the index of refraction outside the laser. With these assumptions, the wave equation for the lateral modes can be rendered dimensionless and written as

$$
\frac{\mathrm{d}^{2} E}{\mathrm{~d} X^{2}}+\left[\Delta+\kappa\left(e^{i 2 X}+e^{-i 2 X}\right)\right] E=0,
$$

where $X$ is the normalized lateral dimension $X=2 \pi x /$ $\Lambda$, and $\Delta$ and $\kappa$ are the normalized eigenvalue detuning and effective index perturbation, respectively:

$$
\Delta=\frac{\Lambda^{2}}{\lambda_{0}^{2}}\left(n_{0}^{2}-\eta^{2}\right), \quad \kappa=\frac{\Lambda^{2}}{\lambda_{0}^{2}} n_{1}^{2} .
$$

We take $E(X)$ as the following sum of two counterpropagating waves:

$$
E(X)=a(X) e^{i X}+b(X) e^{-i X}
$$

When $a$ and $b$ are constant, the wave vector of $E$ satisfies the Bragg condition; since this does not correspond to wave propagation, we must allow $a(X)$ and $b(X)$ to be slowly varying in $X$. When $\kappa=0, a$ and $b$ are simple exponentials and reflect the constant gain under the stripe. When $\kappa \neq 0$, the spatially modulated carrier distribution acts as a grating to couple the counterrunning waves, and $a$ and $b$ are more complicated. By substituting Eq. (4) into Eq. (2), making the usual adiabatic approximation (neglecting $a^{\prime \prime}, b^{\prime \prime}$ ), keeping only the synchronous terms, and setting the slowly varying coefficients of $e^{i X}$ and $e^{-i X}$ equal to zero, we get the following coupled-mode equations in $a$ and $b$ :

$$
\begin{aligned}
2 i a^{\prime} & =(1-\Delta) a-\kappa b, \\
-2 i b^{\prime} & =-\kappa a+(1-\Delta) b .
\end{aligned}
$$

This is a standard eigenvalue problem, whose general solution is

$$
\begin{gathered}
a(X)=A_{+} e^{i \Gamma X}+A_{-} e^{-i \Gamma X}, \\
b(X)=B_{+} e^{i \Gamma X}+B_{-} e^{-i \Gamma X},
\end{gathered}
$$

where $\mathrm{A}_{ \pm}$and $\mathrm{B}_{ \pm}$are complex constants and $\Gamma=\Gamma(\Delta)$ is given by

$$
\Gamma(\Delta)=\frac{1}{2} \sqrt{(1-\Delta)^{2}-\kappa^{2}} .
$$

Strict observation of the slowly varying approximation requires that $|\Gamma| \ll 1$ in the solution; however, we see that reasonable results are obtained even as $|\Gamma| \rightarrow$ 1. Finally, substitution of Eqs. (6) into Eq. (4) gives the general expression for the lateral mode as

$$
\begin{aligned}
E(X)= & {\left[r_{+} e^{i(1+\Gamma) X}+e^{-i(1-\Gamma) X}\right]+r_{-} } \\
& \times \frac{B_{-}}{B_{+}}\left[e^{i(1-\Gamma) X}+r_{+} e^{-i(1+\Gamma) X}\right],
\end{aligned}
$$

where $r_{ \pm}$are the eigenvectors of the system [Eqs. (5)]:

$$
r_{ \pm} \equiv \frac{A_{ \pm}}{B_{ \pm}}=\frac{\kappa}{(1-\Delta) \pm 2 \Gamma(\Delta)} .
$$
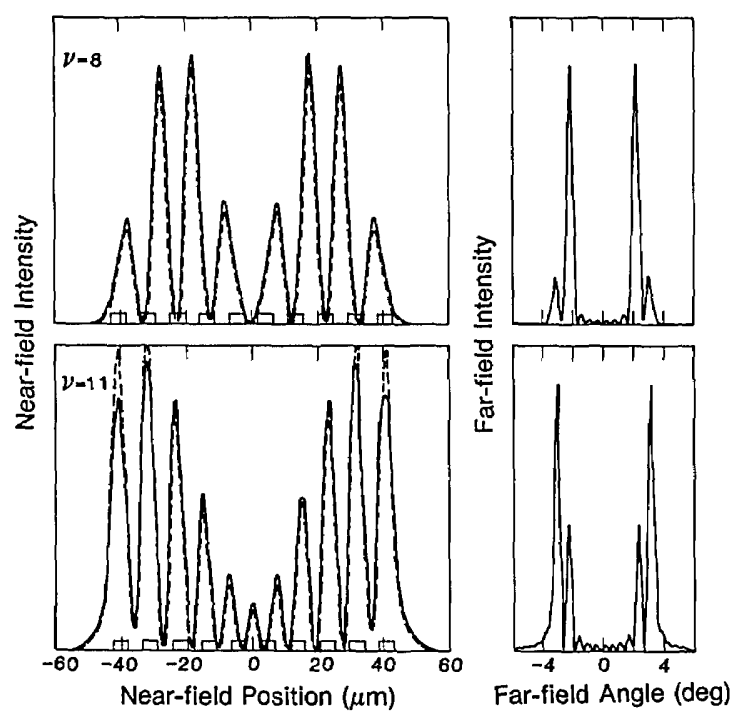

Fig. 3. Near-field and far-field intensities for the $\nu=8$ and $\nu=11$ lateral modes found by numerical solution (solid lines) compared with the analytical solution (dashed lines). 
At this point there are two unknown complex constants, $B_{-} / B_{+}$and the eigenvalue $\Delta$ [or, equivalently, $\Gamma(\Delta)$ ]. Matching our solution [Eq. (8)] for $E(X)$ inside the laser to solutions of the form $E_{1}$ $\exp \left(-i \sqrt{\Delta-\Delta_{0}}\left|X-X_{0}\right|\right)$ outside the laser, where the index is assumed constant, gives the following two boundary conditions:

$$
\left.\frac{E^{\prime}}{E}\right|_{X= \pm X_{0}}= \pm i \sqrt{\Delta-\Delta_{0}}
$$

where $\Delta_{0}$ is the complex constant defined by

$$
\Delta_{0} \equiv \frac{\Lambda^{2}}{\lambda_{0}^{2}}\left(n_{0}^{2}-\bar{n}_{0}^{2}\right)
$$

Equation (10) represents two equations for the two unknowns $B_{-} / B_{+}$and $\Gamma(\Delta)$. For the case of a symmetric device, $B_{-} / B_{+}= \pm r_{+}$, corresponding to even and odd modes, respectively. Since, from Eq. (9), $r_{+} r_{-}=$ 1, Eq. (8) reduces to

$$
\begin{gathered}
E_{\text {even }}(X)=\cos (1-\Gamma) X+r_{+} \cos (1+\Gamma) X \\
E_{\text {odd }}(X)=\sin (1-\Gamma) X-r_{+} \sin (1+\Gamma) X .
\end{gathered}
$$

As $\kappa \rightarrow 0, r_{+} \rightarrow 0$ and we recover the modes of the box waveguide. Thus the second term embodies the distributed feedback and $\Gamma(\Delta)$ gives the detuning from the Bragg wave vector.

To illustrate the result of this approach, we compare the modes of Eqs. (12) with those obtained earlier by direct numerical integration of the wave equation. The appropriate parameters to model the device of Fig. 1 are $\kappa=0.3+i 0.2$ and $\Delta_{0}=-4.0-i 2.67$. We have superimposed the analytical results (dashed lines) onto the numerical results in Figs. 2 and 3. Good agreement is observed for the $\nu=8,9,10$, and 11 modes, for which $|\Gamma| \ll 1$. However, even as $|\Gamma| \rightarrow 1$ the agreement is still reasonable, as illustrated by the $\nu$ $=1$ mode.

As expected, there is a resonance between the $\nu=9$ and $\nu=10$ modes because their lateral wave vectors bracket the Bragg wave vector, $\pi / \Lambda$. The peaks of the near field lie almost exactly under the stripes for the $\nu$ $=10$ mode, while for the $\nu=9$ mode they lie between the stripes. Thus the greatest gain discrimination is achieved between these two modes. Both modes have a half-cosine or first-order envelope, however, being nearly symmetrically displaced from the Bragg wave vector. ${ }^{8}$ Likewise, we expect the $\nu=8$ and $\nu=11$ modes to share second-order envelopes, since together they undergo a gain splitting similar to the $\nu=9$ and $\nu$ $=10$ modes. Figure 3 illustrates that this is indeed the case. Further, the $\nu=1$ mode has nine secondary peaks in the near field (not 10, as would be expected from coupled-mode theory) because it is displaced nine modes from the Bragg wave vector.

In conclusion, we have presented an analytical model that accounts for the lateral modes observed in $N$ stripe semiconductor injection lasers. By making an analytical expansion around the Bragg wave vector, we are able to describe lateral modes of order $\nu=1$ to $\nu=$ $2 N-1$. Modes of higher orders could be accounted for (if they exist) by expansion around integer multiples of the Bragg wave vector. We conclude that the lateral modes of multiple-stripe semiconductor lasers are determined by distributed-feedback resonances of the carrier-induced lateral gain and index perturbation, rather than by superposition of the modes of individual lasers.

The research described in this Letter was conducted in the Department of Applied Physics, California Institute of Technology, Pasadena, California, with the support of the Office of Naval Research and the $\mathrm{Na}$ tional Science Foundation. The authors gratefully acknowledge useful discussions with M. Mittelstein and W. Marshall.

\section{References}

1. E. Kapon, J. Katz, and A. Yariv, Opt. Lett. 10, 125 (1984).

2. J. K. Butler, D. E. Ackley, and D. Botez, Appl. Phys. Lett. 44, 293 (1984).

3. E. Kapon, C. Lindsey, J. Katz, S. Margalit, and A. Yariv, Appl. Phys. Lett. 44, 389 (1984).

4. J. P. Hohimer, G. R. Hadley, and A. Owyoung, Appl. Phys. Lett. 48, 1504 (1986).

5. G. R. Hadley, J. P. Hohimer, and A. Owyoung, Appl. Phys. Lett. 49, 684 (1986).

6. W. K. Marshall and J. Katz, IEEE J. Quantum Electron. QE-22, 827 (1986).

7. J. E. Epler, N. Holonyak, R. D. Burnham, T. L. Paoli, R. L. Thornton, and M. M. Blourke, Appl. Phys. Lett. 47, 7 (1985).

8. H. Kogelnik and C. V. Shank, J. Appl. Phys. 43, 2327 (1972).

9. J. R. Andrews, T. L. Paoli, and R. D. Burnham, Appl. Phys. Lett. 51, 1676 (1987). 